\title{
ESTRATÉGIAS DA GESTÃO PARA IMPLANTAÇÃO DO MODELO DA REDE CEGONHA EM UMA MATERNIDADE PÚBLICA DE CURITIBA*
}

\author{
Marcelexandra Rabelo ${ }^{1}$, Lillian Daisy Gonçalves Wolff², Giseli Campos Gaioski Leal ${ }^{3}$, Márcia Helena de \\ Souza Freire ${ }^{4}$, Silvana Regina Rossi Kissula de Souza ${ }^{5}$, Larissa de Oliveira Peripolli ${ }^{6}$
}

\begin{abstract}
RESUMO: Objetivou-se analisar estratégias adotadas para a implantação das diretrizes da Rede Cegonha, sob a perspectiva de gestores. Trata-se de uma pesquisa exploratória, de abordagem quanti-qualitativa, cuja coleta de dados ocorreu em 2015, mediante entrevistas a três gestores do nível estratégico de uma maternidade de risco habitual em uma capital do sul do Brasil. Utilizou-se o método de análise do Discurso do Sujeito Coletivo com apoio do software Qualiquantisoft ${ }^{\oplus}$. Como resultados, emergiram as categorias, entre outras: Estratégias para implantação do Modelo Assistencial, e Estratégias para reorganização estrutural do modelo gerencial, que foram condizentes à implantação do modelo assistencial preconizado pela Rede Cegonha. Pode-se concluir, sob a luz desta pesquisa, que a proposta da gestão em implantar um modelo humanizado, pautado em evidências científicas, está em processo de construção, com avanços valiosos, empenho das equipes e valorização do profissional impulsionando as ações de mudança, concretizando a efetivação do cuidado em permanente aprimoramento.
\end{abstract}

DESCRITORES: Políticas públicas de saúde; Saúde materno-infantil; Centros de assistência à gravidez e ao parto; Gestão em saúde.

\section{MANAGEMENT STRATEGIES FOR THE IMPLEMENTATION OF THE STORK NETWORK MODEL AT A PUBLIC MATERNITY IN CURITIBA}

\begin{abstract}
The objective was to analyze the strategies adopted for the implementation of the guidelines of the Stork Network from managers' perspective. An exploratory research with a quantitative and qualitative approach was undertaken. The data were collected in 2015 through interviews with three strategic managers from a common-risk maternity hospital in a capital in the South of Brazil. The analysis method of the Collective Subject Discourse was used with the software Qualiquantisoft ${ }^{\circledR}$. The following categories emerged as results, among others: Strategies for the implementation of the Care Model, and Strategies for the structural reorganization of the management model, which lead to the implementation of the care model recommended by the Stork Network In the light of this research, it can be concluded that the management's proposal to implement a humanized care model based on scientific evidence is under construction, with valuable advances, the teams' effort and the valuation of the professional who drives the change actions, putting in practice the continuous improvement of care.
\end{abstract}

DESCRIPTORS: Public health policies; Maternal-infant health; Birthing centers; Health management.

\section{ESTRATEGIAS DE LA GESTIÓN PARA IMPLANTACIÓN DEL MODELO DE LA RED CIGÜEÑA EN UNA MATERNIDAD PÚBLICA DE CURITIBA}

\begin{abstract}
RESUMEN: La finalidad fue analizar estrategias adoptadas para la implementación de las directivas de la Red Cigüeña, bajo la perspectiva de gestores. Se trata de una investigación exploratoria, con aproximación cuanti-cualitativa. Los datos fueron recolectados en 2015, mediante entrevistas con tres gestores del nivel estratégico de una maternidad de riesgo habitual en una capital del sur de Brasil. Fue utilizado el método de análisis del Discurso del Sujeto Colectivo con apoyo delsoftware Qualiquantisoft ${ }^{\oplus}$. Como resultados, emergieron las categorías, entre otros: Estrategias para implantación del Modelo Asistencial, y Estrategias para reorganización estructural del modelo gerencial, que llevaron a la implantación del modelo asistencial preconizado por la RedCigüeña. Se pudo concluir, a la luz de esta investigación, que la propuesta de la gestión en implantar un modelo humanizado, pautado en evidencias científicas, está en proceso de construcción, con avances valiosos, empeño de los equipos y valuación del profesional, impulsando las acciones de cambio, concretizando la práctica del cuidado en permanente perfeccionamiento.
\end{abstract}

DESCRIPTORES: Políticas públicas de salud; Saludmaterno-infantil; Centros de asistencia al embarazo y al parto; Gestión en salud.

*Artigo extraído da dissertação intitulada: "Reorganização da gestão e do modelo de assistência obstétrica em uma maternidade de risco habitual". Universidade Federal do Paraná, 2015.

${ }^{1}$ Enfermeira. Mestre em Enfermagem. Enfermeira Obstétrica na Maternidade Bairro Novo. São José dos Pinhais, PR, Brasil. ${ }^{2}$ Enfermeira. Doutora em Engenharia de Produção. Docente de Enfermagem e do Programa de Pós-Graduação em Enfermagem da Universidade Federal do Paraná. Curitiba, PR, Brasil.

${ }^{3}$ Enfermeira. Mestre em Enfermagem. Docente de Enfermagem da Universidade Federal do Paraná. Curitiba, PR, Brasil. ${ }^{4}$ Enfermeira. Doutora em Saúde Pública. Docente de Enfermagem e do Programa de Pós-Graduação em Enfermagem da Universidade Federal do Paraná. Curitiba, PR, Brasil.

${ }^{5}$ Enfermeira. Doutora em Ciências. Docente de Enfermagem e do Programa de Pós-Graduação em Enfermagem da Universidade Federal do Paraná. Curitiba, PR, Brasil.

${ }^{6}$ Enfermeira Obstétrica. Mestranda em Enfermagem da Universidade Federal do Paraná. Curitiba, PR, Brasil.

Autor Correspondente:

Recebido: $27 / 08 / 2016$

Marcelexandra Rabelo

Finalizado: $11 / 04 / 2017$

Universidade Federal do Paraná

R. Adir Pedroso 777 - 83065-110 - São José dos Pinhais, PR, Brasil

E-mail: marcelexandrar@gmail.com 


\section{INTRODUÇÃO}

A assistência pré-natal de qualidade contribui para a redução de danos à gestante e ao recémnascido. Porém o uso inadequado de tecnologias ou intervenções desnecessárias podem trazer danos para a mãe e recém-nascido ${ }^{(1)}$.

A despeito dos avanços ocorridos na assistência ao parto e nascimento no Brasil, a redução da morbimortalidade materna e infantil permanece um desafio, uma vez que a qualidade da assistência ainda não é satisfatória. Destaca-se a evolução nas políticas públicas brasileiras de saúde da mulher, a partir de iniciativas do Ministério da Saúde. Todavia, as iniciativas parecem ser ainda ineficazes para a necessária mudança no modelo de atenção obstétrica, o qual tem sido rotulado como extremamente intervencionista, expressado nas maiores taxas de cesárea do mundo ${ }^{(1)}$.

Neste cenário, em 2011 o Ministério da Saúde apresentou a estratégia Rede Cegonha, uma rede de cuidados com o escopo de assegurar à mulher o direito ao planejamento reprodutivo; atenção humanizada à gravidez, ao parto, ao puerpério e à criança; o nascimento seguro; bem como o crescimento e o desenvolvimento saudável. Esta rede é classificada em quatro componentes: I. PréNatal; II. Parto e Nascimento, III. Puerpério e Atenção Integral à Saúde da Criança; e IV. Sistema Logístico, incluindo Transporte Sanitário e Regulação ${ }^{(2)}$.

Esta pesquisa desenvolve-se sob as recomendações do Componente II. Parto e Nascimento, sob a responsabilidade das maternidades, o qual inclui a priorização de ações relacionadas às boas práticas de atenção ao parto e nascimento, associadas a investimentos para o aumento e qualificação profissional, assim como à assistência humanizada e de qualidade baseada em evidências científicas e centrada na mulher.

Com a adesão à Rede Cegonha, os gestores das instituições de saúde têm como função conduzir a assistência de acordo com as diretrizes desta estratégia. Mediante o desenvolvimento de ações de planejamento, organização, direção e controle, devem gerenciar recursos humanos, tecnológicos, financeiros, físicos e de informação para o alcance das metas estipuladas no contrato de gestão com as instâncias do Sistema Único de Saúde ${ }^{(2)}$.

A gestão de serviços de saúde tem como prática primordial a otimização do funcionamento dos serviços com vistas ao alcance da eficiência, eficácia e efetividade, por meio do conhecimento e técnicas administrativas. Consequentemente, esta otimização requer a realização de avaliações frequentes e periódicas, como resposta ao desenvolvimento das ações da gestão, e que venham a subsidiar a tomada de decisão ${ }^{(3)}$, visando à reorganização e melhoria dos serviços.

Portanto, além da realização do planejamento estratégico das instituições sob a sua responsabilidade, os gestores devem colocar as estratégias em ação, e estabelecer mecanismos de controle, acompanhamento e avaliação dessas estratégias.

Esta pesquisa pode colaborar com outros serviços de atenção à saúde da mulher que estejam em processo de implantação da Rede Cegonha. Seu objetivo foi analisar as estratégias adotadas pela gestão para a implantação das diretrizes da Rede Cegonha em uma maternidade pública de risco habitual, sob a perspectiva dos gestores.

\section{- MÉTODO}

Trata-se de uma pesquisa exploratória, de abordagem quali-quantitativa, desenvolvida em uma maternidade pública do município de Curitiba, estado do Paraná. A coleta dos dados foi realizada com entrevistas semiestruturadas e individuais, realizadas no segundo semestre de 2015. Os participantes incluídos na pesquisa foram três gestores responsáveis pelo nível estratégico da instituição. As entrevistas foram áudio-gravadas, com duração de uma hora, previamente agendadas conforme disponibilidade e local de preferência do participante assegurando a sua privacidade, tendo como norte a seguinte pergunta: "Conte-nos, na sua perspectiva, como foi a implantação das diretrizes da Rede Cegonha, na maternidade." 
A partir das respostas dos gestores à pergunta norteadora, obteve-se o substrato Discursivo e analisado segundo a proposta do Discurso do Sujeito Coletivo (DSC) com apoio do software Qualiquantisoft ${ }^{\circledast}$ (QQT). Mediante expressões significativas dos discursos, emergiram as Categorias de análise: 1) Dificuldades encontradas para implantação do Modelo Assistencial da Rede Cegonha; 2) Mudança de paradigma; 3) Estratégias da gestão para reorganização estrutural; 4) Estratégias para a implantação do Modelo Assistencial da Rede Cegonha; e 5) Resultados obtidos após a implantação da Rede Cegonha na maternidade, sendo estes os discursos que foram utilizados como recursos metodológicos e construção dos discursos-síntese ${ }^{(4)}$.

Foram respeitados os aspectos éticos e o projeto da pesquisa foi aprovado pelo Comitê de Ética e Pesquisa da Universidade Federal do Paraná, sob o Parecer $n^{\circ}$ 1.126.087. Obteve-se também a aprovação de viabilidade da pesquisa pelo Comitê de Ética da Secretaria Municipal de Saúde de Curitiba-PR.

\section{RESULTADOS}

A partir dos substratos discursivos dos gestores, organizados e analisados de acordo com o Método do Discurso do Sujeito Coletivo, emergiram, entre outras, as categorias: Estratégias para reorganização estrutural do Modelo Gerencial (Quadro 1) e Estratégias para implantação do Modelo Assistencial da Rede Cegonha.

Quadro 1 - Estratégias da Gestão para Reorganização Estrutural do Modelo Gerencial. Curitiba, PR, Brasil, 2015

\begin{tabular}{|c|c|}
\hline Estratégias & Reorganização estrutural \\
\hline Contratação de profissionais & A admissão aconteceu por processo seletivo público. \\
\hline Integração e acolhimento de novos colaboradores & Objetivo de apresentar a filosofia da instituição. \\
\hline Adaptações da estrutura física & $\begin{array}{l}\text { Readequação dos espaços para atendimento e } \\
\text { construção de um jardim externo, para que as } \\
\text { mulheres em trabalho de parto pudessem caminhar. }\end{array}$ \\
\hline Criação do cargo de Enfermeira Obstetra & Concurso e admissão de cinco enfermeiras obstétricas. \\
\hline Criação do colegiado gestor & $\begin{array}{l}\text { Reuniões frequentes entre diretores, gerentes, } \\
\text { coordenadores e profissionais da assistência, com o } \\
\text { propósito de organizar o serviço. }\end{array}$ \\
\hline $\begin{array}{l}\text { Organização da Comissão } \\
\text { acompanhamento da maternidade }\end{array}$ & $\begin{array}{l}\text { Comissão composta por representantes da instituição, } \\
\text { trabalhadores, usuários e conselheiros municipais, } \\
\text { como controle local }\end{array}$ \\
\hline Organização do trabalho na maternidade & Definição dos fluxos, protocolos e rotinas. \\
\hline Indicadores assistenciais & $\begin{array}{l}\text { Elaboração de indicadores, definição de metas e } \\
\text { monitoramento. }\end{array}$ \\
\hline
\end{tabular}

Evidencia-se que a gestão da maternidade reorganizou o seu Modelo Gerencial, com o propósito de adequá-lo às diretrizes propostas pela Rede Cegonha, partindo da contratação e integração de novos profissionais. Neste modelo, foram inseridas no quadro funcional cinco enfermeiras obstétricas, para que iniciassem o atendimento ao parto e nascimento, com cargo e salário específicos.

"[...] Devemos valorizar quem está chegando com novas propostas, com novos conhecimentos, com várias qualidades, várias coisas boas [...] o que a gente sempre buscou trabalhar na maternidade é que cada um tenha o seu espaço, seus limites e sua autonomia, a sempre com limites e respeito".

"[...] sempre falo que ele deve fazer para os outros o que gostaria que fizessem por você, para tratar as pacientes com respeito, carinho e afeto como ele desejaria ser tratado, sem se envolver emocionalmente, mas podemos ser carinhosos dentro do respeito e da ética".

Como estratégia da gestão para reorganizar o Modelo Gerencial, também surgiu a necessidade de 
adaptar a estrutura física, incluindo sala de apoio ao aleitamento materno, sala de práticas integrativas, e sala de descanso do funcionário. De acordo com os gestores, o colaborador é uma ferramenta essencial no trabalho com as pacientes:

"[...] acredito que a nossa maior ferramenta é o colaborador, e a gente sempre trabalha a questão do respeito e da humanização. Quando inauguramos, por exemplo, a sala de práticas, que tem escalda pés, os primeiros que o fizeram foram os funcionários. [...]Nós damos a oportunidade tanto para as mulheres quanto para os nossos funcionários, pensando também no bem-estar deles".

"[...]Também criamos um Colegiado Gestor, que não é só da direção, mas ele é ampliado com a equipe multiprofissional. Se necessário, chamamos outros profissionais para nos colocar as dificuldades, as sugestões com o objetivo de trabalhar melhor".

Em relação à Comissão Permanente de Acompanhamento da Maternidade, os gestores apontam para diversas estratégias que incluem a participação da comunidade, com vista a viabilizar a discussão sobre o que tem sido feito, além de favorecer o feedback da população usuária para o aprimoramento progressivo do serviço:

"[...] criamos também uma espécie de 'orientação' do Conselho Municipal de Saúde, a Comissão de Acompanhamento da maternidade, semelhante a um Conselho Local formado por gestores, trabalhadores, e usuários de abrangência municipal, possibilitando que a gente fosse mostrando nosso trabalho, discutindo as mudanças, e eles trazendo os resultados da comunidade".

Os gestores mencionam também a importância dos indicadores monitorados pela maternidade, os quais são acordados anualmente no contrato de gestão, assim como as metas a serem atingidas e avaliadas:

"[...] Realizamos monitoramento mensal através de indicadores assistenciais e planos de ação a fim de aprimorar nossos serviços. [...] Temos todos os indicadores de boas práticas, acompanhamos as taxas de infecção[...], e tudo que se faz no hospital. Discutimos os dados, no mínimo de dois em dois meses, pra que a gente possa avaliar com o Colegiado Gestor, e ser apresentado também para controle social, para a Secretaria e chefia da unidade [...]".

No Quadro 2 estão descritas as Estratégias da gestão para implantação do Modelo Assistencial dentro das diretrizes a Rede Cegonha.

Quadro 2 - Estratégias da gestão para implantação do Modelo Assistencial. Curitiba, PR, Brasil, 2015 (continua)

\begin{tabular}{|c|c|}
\hline Estratégias & Implantação \\
\hline Elaboração de Protocolos assistenciais & $\begin{array}{l}\text { Capacitação da equipe multidisciplinar com base } \\
\text { nos protocolos desenvolvidos integradamente pelos } \\
\text { profissionais da assistência. }\end{array}$ \\
\hline $\begin{array}{l}\text { Sensibilização das equipes sobre a humanização e a } \\
\text { prática baseada em evidências }\end{array}$ & Treinamentos e cursos. \\
\hline Atuação de enfermeiras obstétricas & $\begin{array}{l}\text { Assistência direta à mulher durante o trabalho de } \\
\text { parto e parto. }\end{array}$ \\
\hline $\begin{array}{l}\text { Implantação efetiva das Boas Práticas de atendimento } \\
\text { ao Parto e Nascimento/OMS* e Rede Cegonha }\end{array}$ & $\begin{array}{l}\text { Práticas baseadas em evidências científicas, } \\
\text { recomendadas mundialmente. }\end{array}$ \\
\hline $\begin{array}{l}\text { Utilização de métodos não farmacológicos para alívio } \\
\text { da dor }\end{array}$ & $\begin{array}{l}\text { Métodos oferecidos às parturientes, como massagem, } \\
\text { banho terapêutico e exercícios na bola suíça, ambiente } \\
\text { silencioso, penumbra e música }\end{array}$ \\
\hline Sala de práticas integrativas & $\begin{array}{l}\text { Espaço adaptado para receber mulheres em trabalho } \\
\text { de parto para relaxamento, com cuidados como } \\
\text { massagem, aromaterapia e escalda pés. }\end{array}$ \\
\hline Acolhimento com classificação de risco & Atendimento classificado por prioridade. \\
\hline $\begin{array}{l}\text { Capacitação e inserção de doulas comunitárias } \\
\text { voluntárias }\end{array}$ & $\begin{array}{l}\text { Curso de formação ofertado pela própria Maternidade } \\
\text { para mulheres da comunidade, com o intuito de as } \\
\text { inserirem no apoio às mulheres em trabalho de parto. }\end{array}$ \\
\hline
\end{tabular}




\begin{tabular}{|c|c|}
\hline Formação profissional & $\begin{array}{l}\text { Residência em enfermagem obstétrica com } \\
\text { sustentação teórico-prática do cuidado obstétrico no } \\
\text { modelo humanizado. }\end{array}$ \\
\hline Educação em saúde: direito à à informação & $\begin{array}{l}\text { Consulta pré natal com a enfermeira da maternidade } \\
\text { (37 semanas de gestação). } \\
\text { Construção do plano de parto. } \\
\text { Oficinas para gestantes e familiares } \\
\text { Consulta de orientações na alta hospitalar, com a } \\
\text { enfermeira. }\end{array}$ \\
\hline $\begin{array}{l}\text { Ações para promoção de vínculo entre profissionais e } \\
\text { gestantes }\end{array}$ & $\begin{array}{l}\text { Ecografia Ecológica (pintura do bebê na barriga da } \\
\text { mãe); Impressão da placenta } \\
\text { - Moldes de barriga (confeccionados com gesso) }\end{array}$ \\
\hline \multirow[t]{3}{*}{ Cuidados Humanizados ao recém-nascido } & Cuidados com o bebê na primeira hora são postergados \\
\hline & $\begin{array}{l}\text { Uso do colete e promoção do Contato pele a pele na } \\
\text { primeira hora }\end{array}$ \\
\hline & Banho tipo Ofurô do recém nascido \\
\hline
\end{tabular}

*OMS - Organização Mundial da Saúde

Em relação à capacitação da equipe, os gestores apontam como estratégias: uma visita técnica dos gestores em um serviço de referência; a participação de enfermeiras obstétricas em curso de aprimoramento no período de 15 dias a um hospital de referência em parto humanizado, promovido pelo Ministério da Saúde; Cursos de Doulas, ofertado pela própria Maternidade para atuação voluntária, de acordo com a filosofia da instituição; capacitação da equipe administrativa; apoio com ênfase no acolhimento; e curso de aleitamento materno para toda a equipe.

"[...] Outra estratégia do Ministério da Saúde foi levar os enfermeiros obstétricos para um curso de imersão e aprimoramento, elas ficaram durante 15 dias no Hospital Sofia Feldman, referência para o parto humanizado. Assim, nós trouxemos e implantamos esses processos de uma forma mais tranquila, porque nós vimos como funcionava lá e adequamos aqui[...]. A partir dessa vivência eu, como gestora, me fortaleci mais ainda, acreditando que é possível desenvolver a autonomia da mulher".

O trabalho em equipe é fundamental para que o planejamento e implementação ocorram de acordo com as diretrizes do Ministério da Saúde e da Secretaria Municipal de Saúde (SMS). No entanto, de acordo com os gestores, a participação dos enfermeiros foi mais intensa no primeiro momento, com o desenvolvimento dos protocolos e rotinas, cujo produto foi entregue aos demais profissionais que não participaram dessa construção.

"[...] Para a Rede Cegonha elaboramos protocolos. O primeiro, sobre parto e nascimento, foi descrito a partir da própria Rede Cegonha, das diretrizes da OMS e das boas práticas [...] O segundo protocolo já avançou bastante e teve uma participação mais efetiva não só do médico, do enfermeiro, do gestor, mas de toda a equipe multiprofissional, o que permitiu mudar o processo de uma forma mais tranquila".

Os gestores dizem que há incentivo por parte da instituição ao trabalho em equipe. E atuação da enfermeira obstétrica, considerando-se sua competência legal e técnico-científica, implica cuidados imprescindíveis na garantia da segurança da mãe e do recém-nascido, além de oferecer cuidado humanizado, pautado em evidências científicas.

"[...] Os profissionais pegam o plantão juntos, já discutem junto um plano para aquela mulher. $O$ partograma é um trabalho em conjunto, participativo. Mas, mesmo no parto que tiver intervenção, não significa que não vai receber o cuidado do enfermeiro[...]".

A educação em saúde é uma questão bastante valorizada como estratégia de gestão, pois quando a mulher e seu acompanhante estão orientados, podem contribuir significativamente com o processo de parturição. Nesta perspectiva, os gestores reconhecem que a equipe colabora para inserir a mulher nas ações desenvolvidas pelos profissionais, como a oficina da gestante, a consulta de 37 semanas, a consulta de alta puerperal, e ainda, com a construção do plano de parto e as práticas integrativas. 
"[...]No final de 2014, a gente implantou a consulta na $37^{\circ}$ semana com o enfermeiro da Maternidade, tendo em vista que a mulher precisa ser participativa no cuidado dela. Porque não adianta mudar o Modelo de Assistência, se a população não acredita nisso. [...] para que ela chegue na maternidade no dia do nascimento mais empoderada[...]"

Apontam os gestores que, para a mudança do Modelo Assistencial e inserção das Boas Práticas de atendimento ao parto, além de profissionais envolvidos com a mudança, faz-se necessária uma comunicação efetiva, pois é imprescindível oferecer informações à mulher desde o período gravídico, a fim de que ela torne-se participativa, autônoma e empoderada nas decisões do seu parto.

"[...]A comunicação é importante, porque às vezes a mulher não sabe dos seus direitos. Cabe a nós profissionais esclarecermos isso! Devemos orientar as mulheres sobre os cuidados que ela pode ter como um todo, desde o Centro Obstétrico até a Sala de Práticas Integrativas. Nós explicamos o que temos para oferecer, conhecemos quais são suas expectativas. Nós trabalhamos com ambiência e não com o ambiente, temos que dar para essa mulher tudo que ela precisa [...].

Reiteram os gestores que é importante promover o protagonismo da mulher desde a gestação, e durante o parto e nascimento, juntamente com o seu acompanhante, a fim de viabilizar sua satisfação quanto ao atendimento.

"[...]Durante o trabalho de parto tem outras práticas que são feitas que auxiliam a paciente e respeitam o momento dela: a bola, a música, a penumbra, conversas diminuídas[...], várias coisas que deixam a paciente mais tranquila".

\section{DISCUSSÃO}

Nos discursos-síntese dos gestores, ficou evidente que o direcionamento das ações da gestão busca a implantação de um novo Modelo de atenção obstétrica. E segundo autores, a condição para que haja mudança do modelo é voltar-se para as relações humanas entre profissionais, a mulher e sua família. Portanto, faz-se essencial entender que a atenção humanizada é relacional(5).

A importância que os gestores da maternidade conferem à sensibilização dos profissionais para um novo Modelo Assistencial, conforme preconizado pela Rede Cegonha, são emitidas nas respostas com destaque à ênfase que atribuíram ao papel fundamental da gestão nesse processo. O modelo tecnocrático é a realidade em vários serviços. Quando o profissional se depara com a demanda da gestão por mudança nesta realidade, pode haver questionamentos e resistência. Compreende-se que a mudança de Modelo Assistencial na obstetrícia requer uma mudança cultural, e não apenas a implantação de ações. Neste sentido, o processo de mudança deve ser desenvolvido em maior tempo, ser baseado em evidências científicas, contar com resultados positivos, e com a satisfação das mulheres atendidas pelo serviço(6).

Acredita-se que para efetivação destas práticas humanizadas são necessários tempo, cuidado e observação contínua, e que a enfermeira obstétrica é a profissional que detém essas características, por desenvolver a essência deste cuidado. E, segundo publicações, as práticas de cuidados obstétricos, preconizadas nas Políticas Públicas, incluem o respeito à privacidade da mulher e a sua escolha acerca do local e posição do parto; o estímulo à deambulação; a informação constante; a utilização de métodos não farmacológicos para alívio da dor, dentre outras ${ }^{(7)}$.

Os gestores ressaltam a importância do acompanhamento e monitoramento dos indicadores assistenciais, o que passou a ser realizado na implantação da Rede Cegonha na maternidade da pesquisa. Segundo pesquisadores, para a obtenção de bons resultados, faz-se necessário o envolvimento e comprometimento por parte dos profissionais. Para tanto, cabe à gestão formar grupos de trabalhos com aptidões específicas, compostos por profissionais da assistência, educação continuada, e líderes do serviço, a fim de que as discussões sejam fundamentadas e resolutivas ${ }^{(8)}$.

Autores aludem que, na democracia, a busca pela cidadania e a garantia dos direitos das usuárias mobilizaram a criação de novas instâncias participativas na gestão do Sistema Único de Saúde (SUS). Citam como exemplos: a gestão participativa; câmaras setoriais; comitês técnicos; grupos de trabalhos; e colegiado gestor, dentre outros. Esses cenários promovem a participação da sociedade, por meio de 
seus representantes, que, somada à participação ativa dos profissionais, tem propiciado respostas e resultados mais imediatos ${ }^{(8)}$.

Foram relatados o desenvolvimento de metas, planos de ação e monitoramento de indicadores, os quais integram a busca pela qualidade dos serviços. No campo relacionado à assistência à saúde, autores definiram qualidade como "a obtenção dos maiores benefícios com os menores riscos ao paciente e ao menor custo", com foco na tríade da gestão de estrutura - processo - resultado. No tocante à segurança do paciente, coloca que o envolvimento do próprio paciente nos serviços de assistência e em suas decisões, como um ator ativo, constitui-se como um elemento fundamental para a qualidade assistencial| ${ }^{(9)}$.

É destacada pelos gestores a inserção da enfermeira obstétrica com atuação no cenário do parto e nascimento. Apontam que estas contribuem significativamente para a mudança do Modelo Assistencial. Reitera-se que as mesmas têm como práticas profissionais o acolhimento e a oferta de métodos não farmacológicos para alívio da dor e cuidado humanizado. Essas práticas foram destacadas nos discursos como utilizadas com frequência na Maternidade, e sua contribuição na implantação das Boas Práticas de atendimento ao parto e nascimento foi evidenciada.

Autores evidenciam que a enfermeira obstétrica tem contemplada em sua formação a filosofia do cuidado humanizado, segundo o recomendado pela OMS. E assim, deve colocar ao dispor das parturientes a atenção profissional específica e qualificada, de forma transversal e integrada. Logo, deve envolver saberes de diversas disciplinas na construção de um cuidado que promova o conforto e autonomia das mulheres. E como estratégia, são apontadas a de incentivo à mulher, a partir de evidências científicas, para o reconhecimento e desenvolvimento de suas próprias habilidades, e a de utilização de técnicas favoráveis à evolução fisiológica do trabalho de parto ${ }^{(10)}$.

Outras ações desenvolvidas pelas enfermeiras e reconhecidas pelos gestores são a realização da Ecografia Ecológica, por meio da qual simula-se a posição do bebê no ventre materno; a confecção do Molde da Barriga por meio do gesso, permitindo a recordação do abdômen grávido; e também a Impressão da Placenta no papel após o parto, como um carimbo real, que simboliza a denominada "Árvore da Vida". Todas estas ações vêm ao encontro dos princípios da humanização, e promovem o vínculo entre a mulher, o acompanhante e o profissional, criando um ambiente agradável e harmonioso.

O modelo humanizado tem como pressupostos o empoderamento da mulher, a promoção da saúde e a garantia dos direitos sexuais e reprodutivos. Diante deste conceito, pode-se entender que a protagonista do parto é a mulher e não mais o profissional ${ }^{(7)}$.

Foram também apontadas, pelos gestores, outras estratégias essenciais, entendidas como de Educação em Saúde, por estarem direcionadas a orientações e informações direcionadas à mulher, a exemplo da oficina da gestante, associada a palestras; consulta com a enfermeira da maternidade no pré-natal (com 37 semanas), com a construção individual do plano de parto; consulta do enfermeiro visando o preparo da usuária para a alta hospitalar.

Estudos mostram que a educação em saúde no ciclo grávido-puerperal é fundamental para a gestante, pois promove maior segurança e minimiza complicações futuras, bem como diminui a frustração e ansiedade maternas, além de proporcionar a valorização das experiências a serem vivenciadas, proporcionando-lhe maior conhecimento e domínio sobre seu corpo, e sobretudo, capacitando-a para participar das decisões acerca de sua gravidez, parto e nascimento ${ }^{(11)}$.

Destaca-se dentre as estratégias assistenciais postas em ação pelos gestores a construção do Plano de Parto Individualizado pela gestante, sob orientação de enfermeiras da instituição. O plano de parto é um documento escrito pela gestante, após ter recebido as orientações sobre o processo de parto e nascimento, e que considera seus valores, desejos e necessidades particulares, contemplando opções pautadas nas Boas Práticas, para direcionar seu atendimento durante o parto ${ }^{(12)}$.

O Programa de Residência em Enfermagem Obstétrica também é entendido como uma estratégia na perspectiva dos gestores, pois considera-se que a presença da formação gera ambientes de discussões, questionamentos e incentivo a pesquisas. Esses contextos promovem críticas reflexivas e crescimento profissional mediante o compartilhamento e a busca integrada pelo conhecimento. 
O Curso de Residência em Enfermagem Obstétrica é visto como promessa de ações educativas com maior potencial para qualificar enfermeiras com habilidades técnicas, que realizam cuidados fundamentados na humanização e em evidências científicas ${ }^{(13)}$.

Por fim, reconhecidamente os protocolos institucionais devem ser pautados nas Políticas Públicas e nas evidências científicas, pois ambos têm como objetivo direcionar as condutas e ações das equipes de saúde.

No entanto, a construção dos processos de humanização e o trabalho baseado em evidências nem sempre estão presentes nas instituições. A explicação para esta dissincronia baseia-se no fato de que tais mudanças requerem, para além da competência técnica de cada profissional, uma disposição humana diferente.

Nesta perspectiva, os profissionais necessitam estar presentes e disponíveis, bem como buscar compreender e respeitar as mulheres e seus acompanhantes, assim como os profissionais de outras áreas, abrindo-se ao diálogo e ao compartilhamento de conhecimentos, de modo a assumirem uma atitude interdisciplinar e eliminarem condutas isoladas ${ }^{(5)}$.

\section{- CONCLUSÃo}

A equipe gestora da Maternidade definiu um novo Modelo Assistencial a ser construído, a partir daí elegeu estratégias efetivas para dar seguimento a esta proposta. Inicialmente, divulgou entre os profissionais as Portarias e Diretrizes, propiciou o desenvolvimento dos protocolos assistenciais, traçou metas e adotou indicadores. Atrelados a estas estratégias, criou o Colegiado Gestor e a Comissão Permanente de Acompanhamento, cujos papéis foram direcionados a agregar discussões para aprimoramento do serviço e atuar no controle social, respectivamente.

Como diferencial marcante desta gestão, destaca-se a atuação efetiva da enfermeira obstétrica na assistência ao parto e nascimento. Ressalta-se que a Direção Geral é conduzida por uma Enfermeira, sendo a Gerente e Coordenadoras enfermeiras obstétricas, cenário este que realça a visão do especialista no processo de gestão, e firma a trajetória condizente com as diretrizes da Rede Cegonha, contribuindo com o cuidado humanizado.

É reconhecido que o empenho dos gestores frente à proposta de mudança do Modelo Assistencial foi fundamental, possibilitando a efetivação dos princípios e recomendações do modelo da Rede Cegonha, no qual a assistência ao parto e nascimento deve ser pautada no cuidado humanizado e em evidências científicas.

Ficou clara a preocupação da gestão com o trabalho da educação em saúde, com o empoderamento das mulheres para o parto e nascimento, mediado por informações baseadas em seus direitos e nas evidências de melhores práticas. Essas ações fortalecem o vínculo entre profissionais e mulheres, promovem maior conhecimento das questões relativas ao parto e nascimento, fomentando o protagonismo e a satisfação das mulheres e seus acompanhantes durante o atendimento.

A oferta do Programa de Residência em Enfermagem Obstétrica na Maternidade foi identificada como valiosa e demonstra a preocupação da gestão em proporcionar às futuras Enfermeiras Obstétricas uma formação qualificada, baseada nos preceitos do SUS, e em evidências científicas.

Finalmente, sob a luz desta pesquisa, acredita-se que a proposta da gestão em implantar um modelo humanizado, pautado em evidências científicas, está em processo de construção, com avanços valiosos, empenho das equipes e valorização do profissional impulsionando as ações de mudança, concretizando a efetivação do cuidado em permanente aprimoramento.

\section{- REFERÊNCIAS}

1. Leal MC, Pereira APE, Domingues RMSM, Theme Filha MM, Dias MAB, Nakamura-Pereira M, et al. Intervenções obstétricas durante o trabalho de parto e parto em mulheres brasileiras de risco habitual.Cad.Saúde Pública. 2014;30(Suppl 1):S17-S32. 
2. Ministério da Saúde (BR). Portaria n.1.459, de 24 de junho de 2011. Institui, no âmbito do Sistema Único de Saúde - SUS - a Rede Cegonha. Diário Oficial da União, Brasília, 2011.

3. Tanaka OY, Tamaki EM. O papel da avaliação para a tomada de decisão na gestão de serviços de saúde. Ciênc. Saúde Colet.2012;17(4):821-8.

4. Lefèvre F, Lefèvre AMC. Pesquisa de representação social: um enfoque qualiquantitativo. Brasília: Liber Livro; 2012.

5. Bruggemann OM, Oliveira ME, Santos EKA. Enfermagem na atenção obstétrica e neonatal. Curitiba: Progressiva; 2011.

6. Ministério da Saúde (BR).Cadernos Humaniza SUS: humanização do parto e do nascimento. Brasília: Ministério da Saúde; 2014.

7. Maia MB. Humanização do parto: política pública, comportamento organizacional e ethos profissional. Rio de Janeiro: Fiocruz; 2010.

8. Harada MJCS,Cunha ICKO. Gestão em enfermagem: ferramentas para uma prática segura. São Paulo: Yendis; 2011.

9. Ministério da Saúde (BR). Agência Nacional de Vigilância Sanitária (ANVISA). Boletim Informativo Segurança do Paciente e Qualidade em Serviços de Saúde. Brasília: Ministério da Saúde/ ANVISA; 2011.

10. de Mattos DV, Vandenberghe L, Martins CA. Motivação de enfermeiros obstetras para o parto domiciliar planejado. Rev enferm UFPE on line. 2014;8(4):951-9.

11. Alencar RM, de Lima SKA, Torres CMG. O processo de educação em saúde da assistência de enfermagem em mulheres gestantes face á realização do pré-natal. Rev. Interfaces. 2014;2(4):1-5.

12. Suárez-Cortés M, Armero-Barranco D, Canteras-JordanaM, Martinez-Roche ME. Uso e influência dos planos de parto e nascimento no processo de parto humanizado. Rev. Latino-Am. Enfermagem. 2015;23(3):520-6.

13. Pereira ALF, Nicácio MC. Formação e inserção profissional das egressas do curso de residência em enfermagem obstétrica. Rev enferm UERJ. [Internet] 2014;22(1) [acesso em 20 nov 2015]. Disponível:http://www.facenf.uerj.br/ v22n1/v22n1a08.pdf. 